\title{
A Rule-Base Approach for WSN Application Development in a Cloud Environment
}

\author{
Saymon Castro de Souza, José Gonçalves Pereira Filho, and Eugênio Fraga Spessimille.
}

\begin{abstract}
The development of applications for wireless sensor networks can be a challenging task, requiring the developer to be knowledgeable of low-level issues from sensor platform, operating system, and programming language together with knowledge of the application domain. One consequence of this scenario is that the WSN programmer receives an overload of demands of development, due to the low participation of the domain expert in the direct definition of rules, events and high-level requirements of the application. This fact can be explained, in part, due to the absence of mechanisms to promote the participation of the domain expert in the process of developing the application. This somewhat complicates the process of universalization of applications based on wireless sensor networks. This paper presents an architecture to face this issue. The proposed solution is based on the integration of rule-based system with sensor-cloud computing environment.
\end{abstract}

Index Terms-Cloud-sensor, wireless sensor network, rule-based system, separation of concern.

\section{INTRODUCTION}

Wireless Sensor Networks (WSN) technology [1], [2] has demonstrated a great potential for industrial, commercial and consumer applications. In the near future, it is expected that WSN will be available virtually everywhere, performing the most diverse tasks of observation and control. However, to achieve the full potential of WSN, the development of applications needs to be facilitated so as to become accessible to users of different fields and disciplines [3]. In general, applications are specifically designed to cater to a specific area, with well defined rules and events. Developers provide the mapping of high- level requirements of domain applications in programs and low-level settings of the base platform of sensor nodes. This fact favors the construction of applications with high degree of coupling between the business logic and hardware platform of the sensor network, a situation that results in a low flexibility of the final product, a platform dependency and maintenance difficulty. The above scenario brings to light three important issues: (i) the developer of WSN applications has to deal with many aspects in the development process of the application, at different levels of abstraction, (ii) participation and interactivity of the user's domain is relatively low in the process, particularly

Manuscript received June 25, 2013; revised August 30, 2013.

Saymon Castro is with the Federal Institute of Espirito Santo, Brazil (e-mail: saymon@ifes.edu.br).

José Gonçalves is with the Department of Computer Science Federal University of Espirito Santo, Vitória, Brazil (e-mail: zegonc@inf.ufes.br).

Eugênio Fraga is with the Federal University of Espírito Santo, Vitória, Brazil (e-mail: eugeniofs@hotmail.com). given the high learning curve of languages and operating systems used in WSN, and (iii) there is a limitation to the use of WSN to the scenario in which it was designed, i.e., the data gathered by WSN are restricted to few applications in the target domain, whereas they could be consumed, in theory, by any applications from different domains interested in the data collected. In relation to (i) and (ii) one of the strategies to address the problem is to promote what in the community of software engineering is known as "separation of concerns" [4], considering in this case two visions: the first refers to the understanding of the details of programming and low-level configurations, communication protocols and sensing of the environment, and the second relates specifically to the rules and application logic, and the domain events. This separation of concerns helps to clearly differentiate the roles of the WSN expert and the domain analyst, which could enhance participation and improve the interactivity of the domain analyst in the application design. Regarding the question raised in (iii), assuming that the data collected by the same network can be shared by a wide range of applications that meet the demands of potentially distinct domains, expanding the universe of target applications can be achieved by decoupling client / server using some strategy for asynchronous communication, for example, the one offered by publish-subscribe mechanisms. Furthermore, in a scenario of rising demand for services based on wireless sensor networks, the cloud computing vision can prepare the solution for future scalability problems.

This paper presents a conceptual architecture that supports the development of WSN applications taking into account the aspects discussed above. The goal of the proposed architecture is to simplify the construction of WSN applications and promote a separation of responsibilities between users, programmers and domain analysts involved in the development process, while allowing to increase the number of applications and the potential areas of use of the data collected by the networks. The paper is structured as follows. Section II presents the proposed architecture. It describes the main functional elements, divided into distinct layers that promote the integration of sensor networks with a rule-based system provided in a cloud computing environment. Section III describes the details of the implementation, Section IV introduces some related work and Section V concludes the paper, presenting the final considerations and future prospects..

\section{Conceptual ARchitecture}

Fig. 1 shows the proposed architecture, which is composed of two distinct layers: the Data Source Layer and Cloud 
Layer. The Data Source layer abstracts the functionality provided by the sensor network, encapsulating aspects of programming and low-level configuration, such as details of the platforms of sensor nodes, specific protocols, operating systems and wireless communication, and interface with the Internet. It also abstracts the source and location of the captured data. It is the layer where the developer with knowledge of the sensor network, i.e., the WSN expert, acts. Each instance of the Data Source Layer includes the sensor network itself, with its nodes and the base station, and the connection to the cloud computing environment, provided by the gateway. The gateway is able to receive the data captured by the sensors and transfer them to a cloud platform in the Internet using a secure channel.

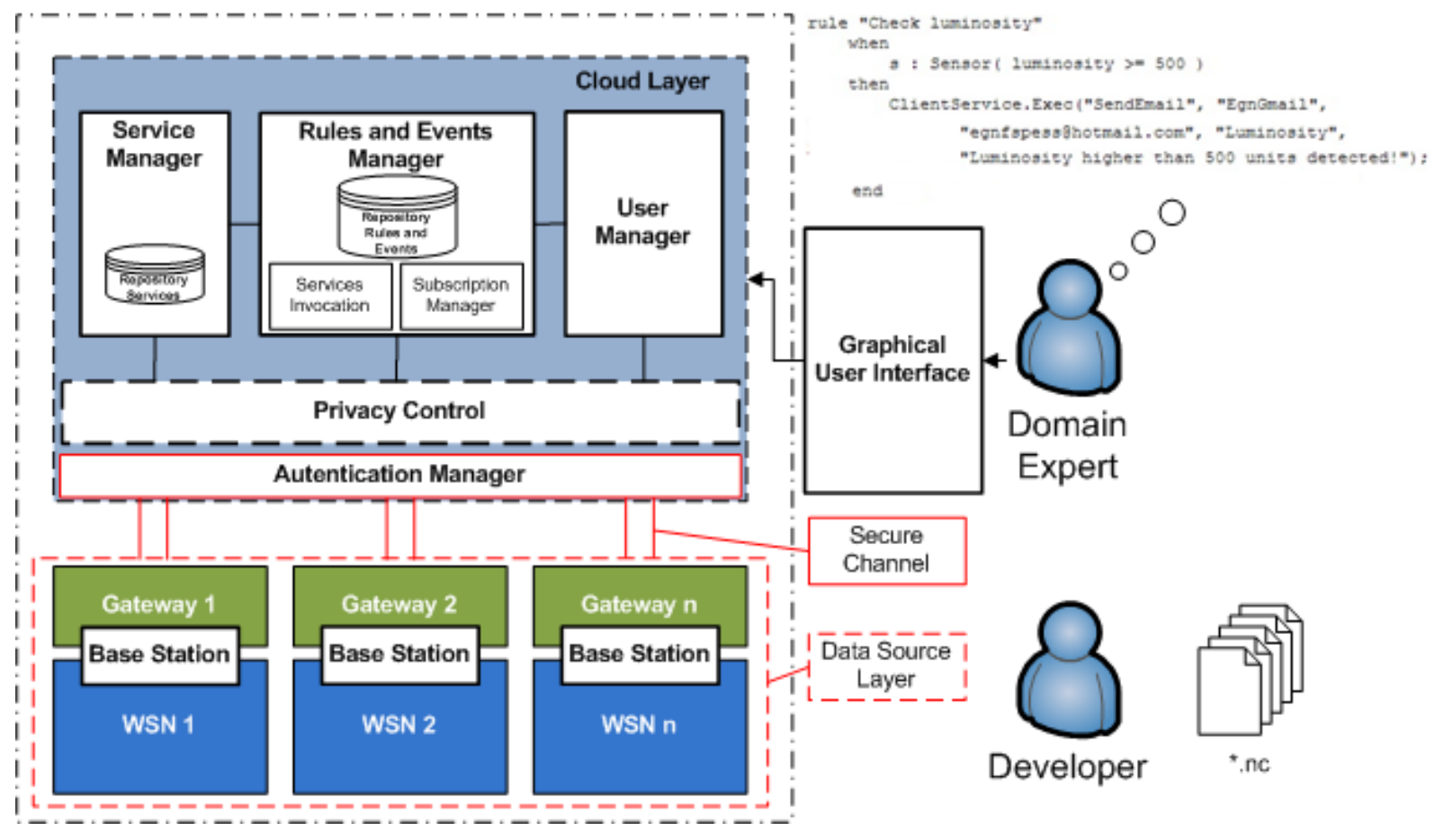

Fig. 1. Conceptual architecture.
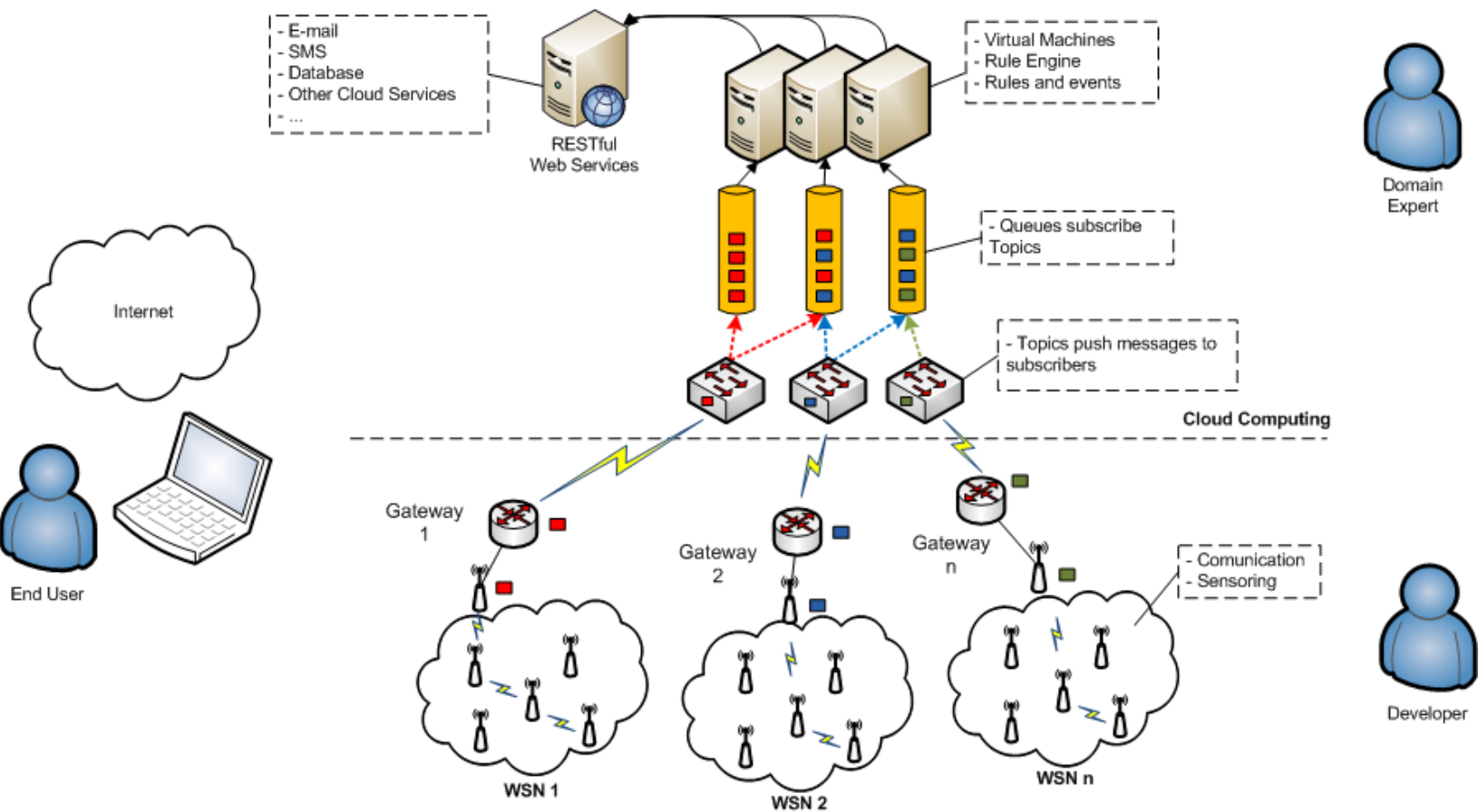

Fig. 2. Implementation.

The Cloud Layer has a set of functions that facilitate the interaction of the domain expert in the process of building the WSN application. This layer abstracts the details of sensing data and network components operation and incorporates essential features for the definition of business rules, applications management and user control. The cloud computing platform only receives sensing data from authorized WSN. Therefore, there must be an element of communication WSN-cloud that has permission to publish the data collected in the WSN environment of cloud 
computing.

The Authentication Manager is responsible for validating credentials and by establishing a secure communication channel between the WSN and the cloud. Access control and permissions settings are provided by the User Manager. The Rules and Events Manager enables subscription to data collected from the WSN, creates rules files in the repository, sets rules and events with a high-level abstraction language, as well as to trigger services available in the repository service. The Service Manager provides facilities for handling services, which may be internal or external to the platform. Consequently, it provides the domain expert with a repository of services, which can be used in the action part of of the rules. A User Interface is provided by the proposed infrastructure to provide access to the elements defined in the Cloud Layer architecture. The interface emphasizes flexibility and portability, and can be deployed on various operating platforms. It should be mentioned that other important requirements, such as privacy and quality of context, are not included in this version of the architecture, which must be the subject of future work.

\section{IMPLEMENTATION}

All The implementation architecture is shown in Fig. 2. According the proposed approach, the WSN expert initially builds the programs that will be installed in the network sensors and gateway, using any programming language suitable for the sensor platform and operating system. The domain expert, in turn, abstracts the WSN details and, through the User Interface, he defines the applications' rules and events. He also defines the networks from which he wants to collect data. For rules and events definition he makes use of the declarative language provided by the infrastructure. Drools [5] was chosen as the rule-based platform. Drools offers a high level abstraction, domain-specific language, called Drools Rule Language (DRL), which provides flexibility and facility to dynamicaly change the rules and events of interest for the application. The application written by analyst domain is represented by a file of rules, which are processed by a virtual machine in a cloud computing environment. To invoke services in the right hand side of the rule, it was developed a client service.

The services were implemented using RESTful Web Services architecture [6], obtaining low coupling and enabling the development of new services to be used by users in the right hand side of the rules. In order to the target WSN start providing data to the cloud it is necessary that the application contained in the gateway starts its execution. In the first run of this application, an encrypted connection is established with the cloud. Credentials are sent to the Authentication Manager so he can performs access validation. At the end of this operation, a secure channel for the transmission of data collected from networks is set. In our implementation, each network transmits the collected data to the base station using a platform-independent data structure, which receives the information via wireless interface and forwards it to the gateway via any appropriate interface (e.g. serial). The gateway, in turn, extracts and encapsulates the information in JSON format [7] and sends them to the respective topic defined in the environment of cloud computing.

The interface Cloud-WSN has been implemented by the base station and the gateway. The application in the gateway generated two components in the implementation process: the Unit Format Data and the Connection Manager. The Unit Format Data receives information from the serial interface, extracts the sensing information contained in the platform-independent data structure, and put such information in JSON format. The Connection Manager establishes a communication channel via https with the cloud computing platform, and the data is sent to the structure of publish / subscribe. Amazon was adopted as a platform for cloud computing and the Amazon Web Services - AWS [8] was used for programmatic interaction with its infrastructure. This way, it was possible to use the services of encrypted connection to the cloud, using authentication credentials. Data sharing was enabled by Simple Notification Services in conjunction with Simple Queue Services [8], generating an architecture publish / subscribe model whose subscription is topic-based. The Subscription Manager enables the receiving of data from the WSN.

It was implemented using a publish / subscribe structure [9], using the subscription-based topic method. The gateways assume the role of publishers and, therefore, associated with each network is instantiated a topic in the cloud. The topic is a virtual representation of the sensed data of a given WSN, being the element of implementation contained in the cloud that allows applications to perform content subscriptions of sensor networks. Signatures can be performed through various protocols, such as email, http, https or queues. The topic receives serialized data and triggers a notification (push) to the subscribers of the topic. This notification message contains metadata and the message that represents the data captured by the WSN. In some scenarios, it is possible that a huge quantity of sensing data may be captured and transmitted, so it is important to have a mechanism to receive data and send them to the applications that have made the necessary subscriptions. In this work, we adopted the queuing model. Queues can be understood as subscribers of topics from which one have interest in receiving data. Each rule-based application has a queue. Message queues arise as the notifications of topics with data arrive from the Data Sources. A thread consumes messages as they arrive on the queue. This thread receives the JSON and transforms it into a JAVA object. To perform the management of the architecture, we developed a web application that allows one to manage the repository of rules and events, perform the control of users, maintain services and define the relationship between the rule based applications and data sources. Thus, the domain applications can consume data from the Data Sources that they are interested in. The User Interface provides the domain analyst with access to various elements of the prototype architecture. For example, it is possible to create, edit and delete users, define access profiles, create, edit and delete files from the repository of rules, and define the users who have developer status access, and which wireless sensor networks one wants to consume data. It is also possible the creation, editing and deletion of services. In the implemented prototype, such services can be built on a server that adopts a 
Rest Web Services architecture. In summary, the User Interface enables the creation of rules, management of signatures for sensing information, and integration services which adopts RESTful architecture in a simple and transparent way.

\section{RELATED WORK}

Ref. [10] presents an overview of architectures for sensor-cloud integration, highlighting the benefits and challenges of putting together these two areas of research. [11] proposes a framework that uses authentication methods and access policies in a cloud computing platform. [12] describes an approach to sensor-cloud integration considering secure sharing of patient data among healthcare profissionals. [13] describe a sensor-cloud virtualization infrastructure that promotes physical sensors sharing among end users. [14] presents a rule-based middleware for wireless sensor networks composed of a set of tools to help in the development process. It created a language for rules definition, called RDL, which is compiled by the execution environment FACTS whose product is a bytecode that is interpreted by the sensor node, thus enabling application interoperability. The architecture proposed in this paper differs from some of the previous work in that it moves the rules and events from inside the WSN to the cloud, allowing for direct participation of the domain expert. Additionally, it also allows the use of internals and externals services in the action part of the rules. Some of the aforementioned studies present approaches in which the rules are present within sensor nodes. However, in scenarios where there are many rules and they change frequently, mentioned approaches may not be appropriate.

\section{CONCLUSION}

This work presented an architecture to support development of applications for wireless sensor networks, based on the use of rule-based system and cloud computing vision,. The proposed approach promotes the division of responsibilities between the WSN programmer expert and the WSN domain expert in building applications. In the proposed architecture, the definition of the rules and the treatment of the events are removed from the sensor nodes which consists of an additional benefit, considering the memory constraints of the motes - and are handled in a cloud computing platform. The prototype implementation made it possible to observe the benefits of the proposed approach. The infrastructure promotes loose coupling of services with regard to the definition of the rules and allowing the development of new services independent of architecture changes. The architecture of publish / subscribe enabled the sharing of sensor data between applications, which subscribe the topics of interest. Thus, some issues have not been addressed in this version of the system, among which we highlight: performance evaluation of implementation, implementation of the privacy component, and the investigation of issues related to the quality of information received from the WSN (QOC - Quality of Context) among other possibilities for improving the infrastructure.

\section{REFERENCES}

[1] J. Yick, B. Mukherjee, and D. Ghosal, "Wireless sensor networks: A survey," Computer Networks, vol. 52, no. 12, pp. 2292-2330, 2008

[2] I. F. Akyildiz, W. Su, Y. Sankarasubramaniam, and E. Cayirci, "Wireless sensor networks: A survey," Computer Networks, vol. 38, no. 4, pp. 393-422, 2002.

[3] T. C. Rodrigues, P. V. Dantas, F. C. Delicato, P. F. Pires, C. Miceli, and L. Pirmez, "Using MDA for building wireless sensor network applications," in Proc. Fourth Brazilian Symposium on Software Components, Architectures and Reuse, SBCARS, pp. 110-119, 2010.

[4] R. J. Mitchell, Managing Complexity in Software Engineering, IEEE, pp. 5, 1990.

[5] M. Bali, Drools Jboss Rules 5.0 Developer's Guide, Packt Pub, 2009.

[6] L. Richardson and S. Ruby, Restful Web Services, O'Reilly Media, 2008.

[7] D. Crockford, The Application/JSON Media Type for Javascript Object Notation (JSON), 2006.

[8] A. E. C. Cloud, Amazon Web Services, Retrieved, June 2013.

[9] P. T. Eugster, P. A. Felber, R. Guerraoui, and A. M. Kermarrec, "The many faces of publish/subscribe," ACM Computing Surveys (CSUR), vol. 35 , no. 2, pp. 114-131, 2003.

[10] A. Alamri, W. S. Ansari, M. M. Hassan, M. S. Hossain, A. Alelaiwi, and M. A. Hossain, "A survey on sensor-cloud: architecture, applications, and approaches," International Journal of Distributed Sensor Networks, vol. 2013, pp. 18, 2013.

[11] K. Ahmed and M. Gregory, "Integrating wireless sensor networks with cloud computing," in Proc. Seventh International Conference on Mobile Ad-hoc and Sensor Networks, 2011.

[12] X. H. Le, S. Y. Lee, P. T. H. Truc, L. T. Vinh, A. M. Khattak, M. Han, D. V. Hung, M. M. Hassan, M. Kim, K. Koo, Y. K. Lee, and E. N. Huh, "Secured wsn-integrated cloud computing for u-life care," in Proc. IEEE Consumer Communications and Networking Conference, 2010.

[13] M. Yuriyama and T. Kushida, "Sensor-cloud infrastructure-physical sensor management with virtualized sensors on cloud computing," in Proc. IEEE 13th International Conference on Network-Based Information Systems, Takayama, Gyfu, Japan, 14-16 Sept. 2010.

[14] K. Terfloth, G. Wittenburg, and J. Schiller, "FACTS-A rule-based middleware architecture for wireless sensor networks," in Proceedings of the 1st International Conference on Communication System Software and Middleware (COMSWARE), 2006.

[15] S. Chen, B. Mulgrew, and P. M. Grant, "A clustering technique for digital communications channel equalization using radial basis function networks," IEEE Trans. on Neural Networks, vol. 4, pp. 570-578, July 1993.

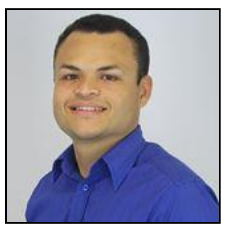

Saymon Castro de Souza was born in 1985 at Vitória, Espírito Santo, Brazil. He is IT Analist at Federal Institute of Espirito Santo (Ifes). Saymon Castro is a master's degree in computer science at Federal University of Espirito Santo (UFES).

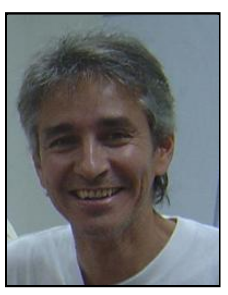

José Gonçalves Pereira Filho is working as associate professor at the Department of Computer Science of the Federal University of Espirito Santo (UFES), in Vitória, Brazil. In 1996, he received Doctoral degree in Electrical Engineering from the University of São Paulo, Brazil. Part of the doctoral research was developed at the University of British Columbia, in Vancouver, Canada. Research topic: multimedia systems.

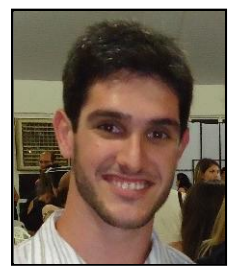

Eugênio Fraga Spessimille was born in 1988 at Vila Velha, Espírito Santo, Brazil. He is a undergraduate student in computer science at University of Espirito Santo (UFES). 